\title{
Morphology of a fibrin nanocoating influences dermal fibroblast behavior
}

This article was published in the following Dove Press journal:

International Journal of Nanomedicine

\section{Julia Pajorova' \\ Marketa Bacakova' \\ Jana Musilkova' \\ Antonin Broz ${ }^{1}$ \\ Daniel Hadraba ${ }^{1,2}$ \\ Frantisek Lopot ${ }^{2}$ \\ Lucie Bacakova'}

'Department of Biomaterials and

Tissue Engineering, Institute of

Physiology of the Czech Academy

of Sciences, Prague, Czech Republic;

${ }^{2}$ Department of Anatomy and

Biomechanics, Faculty of Physical

Education and Sport, Charles

University, Prague, Czech Republic
Correspondence: Julia Pajorova Institute of Physiology, Academy of Sciences of the Czech Republic, Videnska 1083, Prague 4, 14220, Czech Republic Tel +420296443765

Email julia.pajorova@fgu.cas.cz
Background: Our study focuses on the fabrication of appropriate scaffolds for skin wound healing. This research brings valuable insights into the molecular mechanisms of adhesion, proliferation, and control of cell behavior through the extracellular matrix represented by synthetic biodegradable nanofibrous membranes coated by biomolecules.

Methods: Nanofibrous polylactic acid (PLA) membranes were prepared by a needle-less electrospinning technology. These membranes were coated with fibrin according to two preparation protocols, and additionally they were coated with fibronectin in order to increase the cell affinity for colonizing the PLA membranes. The adhesion, growth, and extracellular matrix protein production of neonatal human dermal fibroblasts were evaluated on the nanofibrous membranes.

Results: Our results showed that fibrin-coated membranes improved the adhesion and proliferation of human dermal fibroblasts. The morphology of the fibrin nanocoating seems to be crucial for the adhesion of fibroblasts, and consequently for their phenotypic maturation. Fibrin either covered the individual fibers in the membrane (F1 nanocoating), or covered the individual fibers and also formed a fine homogeneous nanofibrous mesh on the surface of the membrane (F2 nanocoating), depending on the mode of fibrin preparation. The fibroblasts on the membranes with the F1 nanocoating remained in their typical spindle-like shape. However, the cells on the F2 nanocoating were spread mostly in a polygon-like shape, and their proliferation was significantly higher. Fibronectin formed an additional mesh attached to the surface of the fibrin mesh, and further enhanced the cell adhesion and growth. The relative gene expression and protein production of collagen I and fibronectin were higher on the F2 nanocoating than on the F1 nanocoating.

Conclusion: A PLA membrane coated with a homogeneous fibrin mesh seems to be promising for the construction of temporary full-thickness skin tissue substitutes.

Keywords: nanofibers, fibrin, nanocoating, dermal fibroblasts, extracellular matrix synthesis, skin substitute, polylactic acid

\section{Introduction}

Fibrin is a biomolecule that is physiologically formed after a skin injury. It plays an important role during the healing process of a skin wound. After a tissue injury, the coagulation cascade is activated, and as a result, water-soluble fibrinogen is converted into insoluble fibrin. The fibrin forms a network of fibers that stabilizes the platelet plug by binding platelets to fibrin fibers. The platelets secrete growth factors, mainly platelet-derived growth factor and transforming growth factor beta. These growth factors stimulate the fibroblasts to migrate into the wound, proliferate, and produce extracellular matrix (ECM) proteins, mainly collagen I and fibronectin (Fn). The synthesized extracellular proteins finally replace the fibrin network. ${ }^{1,2}$ Through the cell adhesion receptors, the fibrin binds the fibroblasts and the other cell types participating in the 
wound healing process, such as endothelial cells, smooth muscle cells, leukocytes, fibroblasts, and keratinocytes. ${ }^{3}$ The fibrin molecule also binds growth factors and ECM proteins Fn and vitronectin, which are important for cell adhesion. ${ }^{1}$

Fibrin is often applied in regenerative medicine to support wound healing. To accelerate wound healing, fibrin is mostly used in the form of a sealant, ${ }^{4,5}$ a gel, ${ }^{6-8}$ a glue,,${ }^{9,10}$ or microbeads. ${ }^{11}$ It is directly applied into the wound and is seeded with skin cells. However, the fibrin is relatively rarely combined with other supporting substrates, although this could improve the stability of various matrices. In our previous studies, we fabricated fibrin-coated polylactic acid (PLA) or polylactide-co-glycolide (PLGA) nanofibrous membranes, and we observed a positive influence of fibrin on the behavior of dermal fibroblasts. Moreover, the coated nanofibrous membrane showed good mechanical properties for withstanding the traction forces generated by fibroblasts. ${ }^{12,13}$

In this study, we prepared electrospun PLA membranes with fibrin nanocoatings of various structures. The first nanocoating only coated each individual membrane fiber. The second nanocoating covered the individual fibers, and in addition it formed a fine nanofibrous mesh on top of the membrane and filled the pores between the fibers. In addition, we attached Fn to the fibrin nanocoating to enhance cell adhesion. ${ }^{13}$ The purpose of this study was to compare the behavior of human dermal fibroblasts on fibrin-coated membranes depending on the different structure of the fibrin nanocoating. We observed the fibroblast adhesion, spreading, proliferation, and synthesis of ECM proteins - collagen I and Fn. It is known that fibroblasts secrete ECM proteins during wound healing, mainly collagens of type I and type III, the most abundant proteins in skin dermis. The synthesis of extracellular collagen is enhanced by 2-phospho-L-ascorbic acid trisodium salt (AA). AA plays an important role in the formation of stable mature collagen with a triple-helix structure. AA serves as a cofactor for enzymes catalyzing the synthesis of hydroxyproline and hydroxylysine. These amino acids are important for intermolecular crosslinking in the collagen structure. ${ }^{14,15}$ In our previous study, we reported that dermal fibroblasts cultivated on fibrin-coated membranes deposited type I extracellular collagen on the surface of the membrane. In addition, fibrin significantly increased the synthesis of extracellular collagen, as determined by an increasing expression of mRNA and an increasing amount of protein deposited on the membrane. ${ }^{12}$ In a subsequent study, we observed that fibrin increased the synthesis of another extracellular protein, Fn. ${ }^{13}$

In recent times, nanostructured materials have been applied increasingly in the fabrication of tissue substitutes. In our studies, we also use biodegradable nanofibrous membranes for cell cultivation, because these materials can provide more support for cell adhesion, proliferation, and differentiation than flat and microstructured surfaces. ${ }^{16}$ The nanostructure of the materials mimics the nanoarchitecture of the natural ECM, and thus enhances the cell-material interaction. Cell adhesion is mediated through cell integrin receptors, which bind ECM proteins. For successful cell adhesion, the ECM proteins should be adsorbed on the substrate from the body fluid or from the cell culture medium in a sufficient amount, and particularly in an appropriate spatial conformation. The spatial conformation of ECM molecules is important for their interaction with cell adhesion receptors. Nanostructured materials are able to adsorb ECM adhesion-mediating molecules in an appropriate spatial conformation, and thus they enable the cells to attach and spread on the substrate. ${ }^{16,17}$

\section{Materials and methods Preparation of nanofibrous membranes}

Nanofibrous PLA membranes were prepared by the electrospinning process, as was described in our earlier paper. ${ }^{12}$ PLA Ingeo $^{\mathrm{TM}}$ Biopolymer 4032D (NatureWorks, Minnetonka, MN, USA) was dissolved in chloroform. Dichloroethane and ethyl acetate were added into the PLA solution to a final concentration of $7 \mathrm{wt} \%$ of PLA. Tetraethylammonium bromide was added to the PLA solution to make the polymer solution electrically conductive.

A PLA nanofibrous membrane was prepared using Novel Nanospider needle-free electrospinning technology (NS Lab 500, Liberec, Czech Republic). The process was carried out under the following conditions: electrode distance: $180 \mathrm{~mm}$; voltage: $60-10 \mathrm{kV}$; the spinning electrode rotated at $4 \mathrm{rpm}$; relative humidity: $25 \%-30 \%$, and room temperature. The membranes were prepared at a fiber density of $17 \mathrm{~g} / \mathrm{m}^{2}$, that is, the area weight of the prepared nanofibers.

\section{Preparation of fibrin nanocoatings with attached Fn}

The fibrin nanocoatings on the PLA nanofibrous membranes were formed by activation of water-soluble human fibrinogen (341576; EMD Millipore, Billerica, MA, USA) with human thrombin (T6884; Sigma-Aldrich Co, St Louis, MO, USA), in accordance with a previously published work. ${ }^{18}$

Two different types of fibrin nanocoatings were prepared on the membranes. Ten micrograms per milliliter of fibrinogen diluted in Tris buffer (consisting of $50 \mathrm{mM}$ Tris- $\mathrm{HCl}$, $100 \mathrm{nM} \mathrm{NaCl}$, and $2.5 \mathrm{mM} \mathrm{CaCl}_{2}$ ) was adsorbed on the membrane for 1 hour. After rinsing with Tris buffer, the adsorbed 
fibrinogen was activated with thrombin $(2.5 \mathrm{U} / \mathrm{mL}$ in Tris buffer) for 15 minutes. These first two preparation steps were the same for both types of fibrin nanocoatings. The subsequent steps differed according to the type of nanocoating. The first type of fibrin nanocoating covered the individual fibers in the membrane (F1 nanocoating). The second type of fibrin nanocoating covered the individual fibers in the membrane and, in addition, it formed a fine homogeneous fibrin mesh on the surface of the membrane and filled the pores between the membrane fibers (F2 nanocoating).

When preparing the F1 nanocoating, the thrombin-activated membranes were incubated in Tris buffer for 30 minutes in order to wash out the unreacted thrombin. Then a solution of $200 \mu \mathrm{g} / \mathrm{mL}$ of fibrinogen in Tris buffer and $0.5 \mathrm{U} / \mathrm{mL}$ of antithrombin III (Chromogenix, Milano, Italy) in deionized water was added to the membranes for 1 hour to form a stable fibrin nanocoating. The antithrombin III blocked the rest of the unreacted thrombin to prevent the formation of a three-dimensional (3-D) fibrin gel. The F2 nanocoating was prepared directly after thrombin activation, without the incubation step in Tris buffer, that is, without washing out the unreacted thrombin. In this case, the unreacted thrombin reacted with the ambient fibrinogen and a fine nanofibrous fibrin mesh was formed.

To enhance fibroblast adhesion, we attached Fn to the surface of fibrin nanocoating. Human Fn powder (11051407001; Roche, Basel, Switzerland) was dissolved in deionized water at a concentration of $1 \mathrm{mg} / \mathrm{mL}$. The Fn solution with a final concentration of $50 \mu \mathrm{g} / \mathrm{mL}$ in phosphate-buffered saline (PBS; Sigma-Aldrich Co) was added to the samples and was incubated overnight at $4^{\circ} \mathrm{C}$. The samples were then rinsed twice with PBS.

\section{Membrane puncture testing}

The wet pristine membrane and the membrane with an F1 or F2 nanocoating were tested using the puncture testing protocol, described in detail in our earlier study. ${ }^{13}$ Six samples were used for each experimental group. In brief, the samples were placed in a customized holder and were punctured with a spherical cup probe at a constant speed of $0.3 \mathrm{~mm} / \mathrm{s}$ (stepper motor PD283-1021; Trinamic, Hamburg, Germany) while the force was being recorded $(1,000 \mathrm{~Hz}$, Force sensor Kistler 9203; Kistler, Winterthur, Switzerland). The structural integrity of the membranes was optically checked at microscale level, using reflectance confocal microscopy (xyt scan, C PLAN magnification $4.0 \times / 0.10$ numerical aperture [NA] dry, $256 \times 256$ pixels, pixel size $3.6 \mu \mathrm{m}$, Leica TCS SPE coupled to Leica DM2500 [Leica Microsystems, Wetzlar, Germany]) and, at macroscale level, by a digital camera $(1,504 \times 880$ pixels, pixel size $0.015 \mathrm{~mm}$, M5; Integrated Design Tools, Inc., Hitchin, UK).
As the membranes were from a single batch, the evaluation was made using Hooke's law, that is, $F=-k x$, where $F$ is the detected force, $x$ is the membrane extension, and $k$ represents the stiffness of the membrane. In addition, the maximum force $F_{\max }$, the extension at maximum force $x_{F \max }$, and the work $W$ that was necessary to completely tear the membrane were also calculated for a statistical comparison (Matlab 2017; Mathworks, Natick, MA, USA).

\section{Cell culture}

Before the fibrin nanocoating was prepared and the membranes were seeded with cells, the nanofibrous membranes were fixed in Cell Crown inserts (Scaffdex Ltd, Tampere, Finland) in order to prevent the sample floating in the cell culture medium. The samples were sterilized in $70 \%$ ethanol for 30 minutes and were rinsed in sterilized deionized water for 2 days. Then the samples were inserted into the wells of 24-, 12-, or 6-well plates (TPP Techno Plastic Products AG, Trasadingen, Switzerland).

The membranes were seeded with neonatal human dermal fibroblasts, purchased from Lonza (Basel, Switzerland), in passages 2-6, at a density of 10,000 cells $/ \mathrm{cm}^{2}$. The cells were cultivated in DMEM (Sigma-Aldrich Co) with 10\% of fetal bovine serum (FBS; Sebak GmbH, Aidenbach, Germany) and $40 \mu \mathrm{g} / \mathrm{mL}$ of gentamicin (Lek, Ljubljana, Slovenia). The cells were incubated for various time intervals, depending on the given experiment, in a humidified atmosphere with $5 \%$ of $\mathrm{CO}_{2}$ in the air. Polystyrene (PS) culture wells were used as a reference material. On day 1 after cell seeding, the culture medium was supplemented with AA at a concentration of $50 \mu \mathrm{g} / \mathrm{mL}$ in order to stimulate the cells to synthesize extracellular collagen.

\section{Morphology of the fibrin nanocoatings}

The morphology of the fibrin F1 and F2 nanocoatings (with or without attached Fn) was studied using fluorescence confocal microscopy. The different fibrin morphology of the F1 and F2 nanocoatings was immunofluorescence stained on freshly prepared cell-free samples and on samples with cells cultivated on membranes for 1, 3, and 7 days. A noncoated membrane was used as a control sample to evaluate possible nonspecific binding of the primary and secondary antibodies.

The membranes were treated with $1 \%$ bovine serum albumin in PBS (Sigma-Aldrich Co) for 20 minutes, and then with 1\% Tween (Sigma-Aldrich Co) in PBS for 20 minutes at room temperature to block nonspecific binding sites. Then the samples were incubated overnight at $4^{\circ} \mathrm{C}$ with primary antibody against human fibrinogen (A0080, polyclonal 
rabbit antibody; Dako Denmark A/S, Glostrup, Denmark) or with Fn (F0791, mouse monoclonal antibody; SigmaAldrich Co), diluted in PBS in a ratio of 1:200. On the next day, the samples were rinsed with PBS and were incubated with secondary antibodies goat anti-rabbit or goat antimouse $\mathrm{F}\left(\mathrm{ab}^{\prime}\right) 2$ fragments of immunoglobulin $\mathrm{G}(\mathrm{IgG}$; $\mathrm{H}+\mathrm{L})$ conjugated with Alexa Fluor ${ }^{\circledR} 488$ (A11070 and A11017, Molecular Probes, Thermo Fisher Scientific, Waltham, MA, USA). The incubation time was 1 hour at room temperature in the dark, and the dilution was 1:400 in PBS.

The fibrin-coated membranes with attached Fn were simultaneously stained for fibrin and Fn on the same sample. In this case, the samples were incubated with a primary antibody against fibrinogen overnight at $4{ }^{\circ} \mathrm{C}$. On the next day, after the samples had been rinsed with PBS, the primary antibody against Fn was added to the samples for 3 hours. Both primary antibodies were diluted in a ratio of 1:200. Finally, the samples were incubated with secondary antibody goat anti-rabbit $\mathrm{F}\left(\mathrm{ab}^{\prime}\right) 2$ fragment of $\operatorname{IgG}(\mathrm{H}+\mathrm{L})$ conjugated with Alexa Fluor 488 and then with secondary antibody goat anti-mouse $\mathrm{F}\left(\mathrm{ab}^{\prime}\right) 2$ fragment of $\operatorname{IgG}(\mathrm{H}+\mathrm{L})$ conjugated with Alexa Fluor 633 (A11070 and A21053, Molecular Probes, Thermo Fisher Scientific). The incubation time for both secondary antibodies was 1 hour in the dark, and the dilution was 1:400 in PBS.

The samples were scanned on a Leica TCS SPE DM2500 upright confocal microscope, magnification 40×/1.15 NA oil, and on a Leica TCS SP8, objective HCX PL APO, magnification $40 \times / 1.25-0.75$ NA Oil CS. The signal intensity profiles of the immunofluorescence-stained fibrin nanocoating with attached Fn were plotted using data obtained by the "Plot Z-axis Profile" function in Fiji (ImageJ) open-source image analysis software. ${ }^{19}$ This software function plots the average image plane intensity in a given color channel versus the $z$-axis (depth) of a confocal $z$-stack image.

\section{Cell spreading}

The spreading and the shape of the cells were evaluated by staining the cell F-actin cytoskeleton and the cell nuclei. The F-actin was stained with phalloidin conjugated with tetramethylrhodamine fluorescent dye (Sigma-Aldrich Co), and the cell nuclei were stained with Hoechst \#33258 (Sigma-Aldrich Co) for 1 hour at room temperature in the dark. Both dyes were diluted in PBS to concentration $5 \mu \mathrm{g} / \mathrm{mL}$. Before the cells were stained with fluorescent dyes, they were rinsed in PBS and were fixed with $-20^{\circ} \mathrm{C}$ cold ethanol for 10 minutes. The images were taken using a Leica TCS SPE DM2500 upright confocal microscope using magnification 40×/1.15 NA oil.

\section{Cell mitochondrial activity}

The activity of cell mitochondrial enzymes was measured on days 1,3 , and 7 after cell seeding as a marker of cell proliferation and viability. The metabolic activity was determined by a CellTiter $96^{\circledR}$ AQueous One Solution Cell Proliferation Assay (MTS; Promega Corporation, Madison, WI, USA). The membrane samples were moved into fresh cell culture wells to avoid the influence of cells adhered to the bottom of the well. The assay was performed according the manufacturer's protocol. The formazan dye produced by the cells after 3 hours of incubation was quantified by measuring the absorbance using a VersaMax ELISA Microplate Reader spectrophotometer (Molecular Devices Corporation, Sunnyvale, CA, USA). The absorbance was measured with wavelength $490 \mathrm{~nm}$ and with reference wavelength $650 \mathrm{~nm}$. Three independent samples were used for each experimental group and time interval. The experiment was performed three times.

\section{Immunofluorescence staining of extracellular collagen I and Fn}

The extracellular collagen I and Fn deposited by the cells on the surface of the membrane were immunofluorescently stained on days 4,7 , and 14 after cell seeding. The samples were rinsed with 5\% FBS in PBS. The membranes were then incubated in primary antibodies against collagen I (C2456, mouse monoclonal antibody; Sigma-Aldrich Co) or Fn (F0791, mouse monoclonal antibody; Sigma-Aldrich Co) diluted in PBS in a ratio of 1:200 for 30 minutes in ice. After the cells were rinsed with 5\% FBS in PBS, they were fixed for 20 minutes with $2 \%$ paraformaldehyde dissolved in PBS with $5 \%$ of FBS. The samples were rinsed with PBS and were incubated in 1\% FBS in PBS to block nonspecific binding sites for 20 minutes. The solution of secondary antibody antimouse $\mathrm{F}\left(\mathrm{ab}^{\prime}\right) 2$ fragments of $\operatorname{IgG}(\mathrm{H}+\mathrm{L})$ conjugated with Alexa Fluor 488 (Molecular Probes, Thermo Fisher Scientific) was diluted 1:400 in 1\% FBS in PBS. This solution was added to the sample for 2 hours in the dark. After being rinsed with PBS, the cell nuclei were stained with Hoechst \#33342, which penetrates through the nonpermeabilized cell membrane. Images were taken using a Leica TCS SPE DM2500 upright confocal microscope, magnification $40 \times / 1.15$ NA oil.

\section{Real-time polymerase chain reaction (PCR)}

Real-time PCR was performed to investigate the relative mRNA expression of ECM proteins, collagen I and Fn, in the dermal fibroblasts. The cells were harvested on days 4 and 7 of cultivation on the modified PLA membrane. The total RNA was isolated using the Total RNA Purification 
Micro Kit (35300; Norgen Biotek, Thorold, ON, Canada), according to the manufacturer's protocol. The cells were rinsed with PBS and the membrane was placed into a $1.5 \mathrm{~mL}$ tube filled with RNA lysis solution enriched with mercaptoethanol (1\%).

Reverse transcription was performed using the Omniscript Reverse Transcription Kit (205113; Qiagen, Hilden, Germany) and random hexamers (New England Biolabs, Inc, Ipswich, MA, USA) and was carried out according to the manufacturer's protocol.

The mRNA level was quantified using $5 \times$ HOT FIREPol Probe qPCR Mix Plus (ROX) (08-36-00001; Solis BioDyne, Tartu, Estonia) and by TaqMan Gene Expression Assays (4331182; Thermo Fisher Scientific), labeled with FAM reporter dye specific to human collagen I COL1A1 (Hs00164004_m1) or specific to human Fn FN1 (Hs01549976_m1) as a target gene, and beta $(\beta)$-actin ACTB (Hs01060665_g1) as a reference gene. The experiments were performed in duplicate. The final reaction volume was $20 \mu \mathrm{L}$ of solution. The mRNA expression level was determined using the Viia ${ }^{\mathrm{TM}} 7$ Real-time PCR System (Applied Biosystems ${ }^{\mathrm{TM}}$; Thermo Fisher Scientific) in a 96-well optical reaction plate. The amplification conditions consisted of uracil-DNA glycosylase incubation at $50^{\circ} \mathrm{C}$ for 2 minutes and initial DNA polymerase enzyme activation at $95^{\circ} \mathrm{C}$ for 10 minutes, followed by 40 cycles of denaturation at $95^{\circ} \mathrm{C}$ for 15 seconds and annealing/extension at $60^{\circ} \mathrm{C}$ for 1 minute.

Two independent samples were used for each experimental group and time interval. The experiment was performed five times. Expression values were obtained from $\mathrm{Ct}$ numbers. The relative gene expression was calculated as $2^{-\Delta \mathrm{Ct}}$.

\section{Statistics}

The data were presented as mean \pm either standard deviation or standard error of the mean. Statistical significance was evaluated using analysis of variance with the Tukey post hoc test for pairwise comparison. Values of $p \leq 0.05$ were considered as significant.

\section{Results}

\section{The morphology and the mechanical properties of two different types of fibrin nanocoatings on a PLA membrane}

The fibers of the PLA membrane were randomly oriented and mostly straight. The diameter of the fibers ranged from tens of nanometer to $>1 \mu \mathrm{m}$. The average fiber diameter was $>300 \mathrm{~nm}$, as we had measured in our earlier studies. ${ }^{12,13}$

In accordance with the protocol for fibrin preparation, fibrin either covered only individual fibers in the PLA membrane (Figure 1, F1) or covered individual fibers and in addition formed a fine homogeneous nanofibrous mesh on the surface of the membrane (Figure 1, F2). In our previous study, fibrin did not form a homogeneous mesh on the whole surface of the membrane. ${ }^{12}$ Fn (Figure 2, red) was adsorbed especially on top of the fibrin mesh (Figure 2, green) and formed an additional nanofibrous mesh (Figure 1, F2+Fn). Fn was also adsorbed on the fibrin of the F1 nanocoating (Figure 1, F1+Fn); however, it was hardly detectable by immunofluorescence microscopy. The stability of the fibrin nanocoatings on the PLA membrane was proved in our previous study. ${ }^{12}$ The morphology of the fibrin nanocoating without seeded cells remained almost unchanged after 7 days in the cell cultivation conditions, while it was progressively degraded on the membranes with cells.

Furthermore, the way in which the fibrin nanocoating had been prepared had a minor effect on the mechanical properties of the PLA membrane. Mechanical puncture testing returned very similar results for all three groups (Table 1). The null hypothesis was rejected only for $x_{F m a x}$, as this parameter decreased significantly for the F1 nanocoating compared to pristine $(p$-value $=0.025)$. By contrast, the other parameters did not differ ( $p$-value for $k=0.599$, $F_{\text {max }}=0.376$ and $W=0.417$ ); however, there was an evident trend toward increasing resistance against mechanical loading for the F2 nanocoating.

\section{Cell adhesion, spreading, and metabolic activity}

The washout step of the remaining unreacted thrombin during the preparation of the fibrin nanocoatings was determined as the crucial point for the formation of a nanofibrous homogeneous mesh on the surface of the membrane (F2 nanocoating), and also for the fibrin nanocoating covering individual fibers of the membrane (F1 nanocoating). Different behavior of human dermal fibroblasts was observed on these two different types of fibrin nanocoating. The number of adhered fibroblasts on the F1 nanocoating was lower, and the cells remained in their typical spindle-like shape (Figure 1, F1). However, the number of adhered fibroblasts on the F2 nanocoating was higher, and the cells tended to adopt a polygon-like shape (Figure 1, F2). As was mentioned above, Fn formed a secondary nanofibrous mesh (Figure 2, red) on the surface of the initially developed fibrin mesh (Figure 2, green) and enhanced the cell adhesion on both types of fibrin nanocoating (Figure 1, F1/F2+Fn). It was observed that fibroblasts (Figure 2, blue and magenta) adhered preferentially on top of Fn mesh (Figure 2, red). Moreover, the shape of the 
Without fibroblasts In PBS
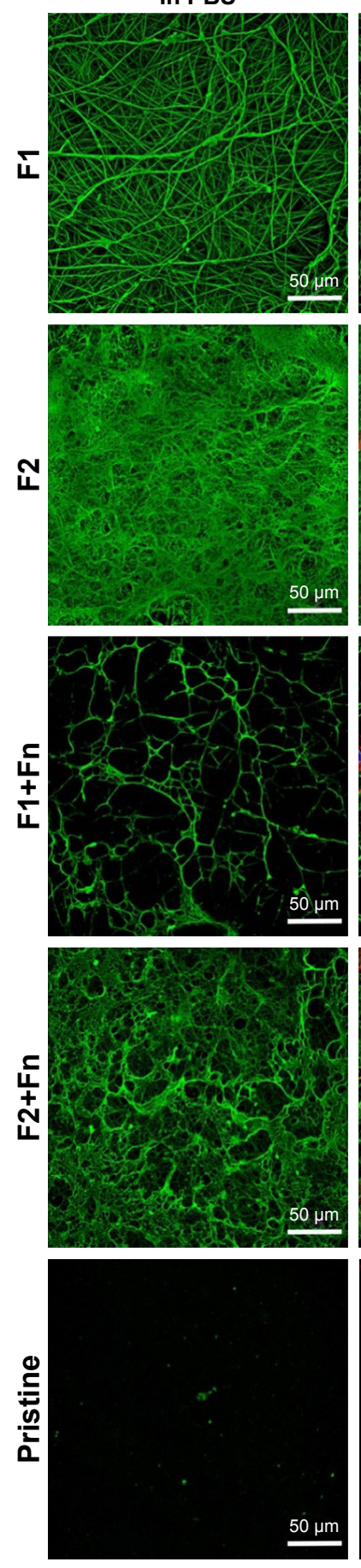

Day 1
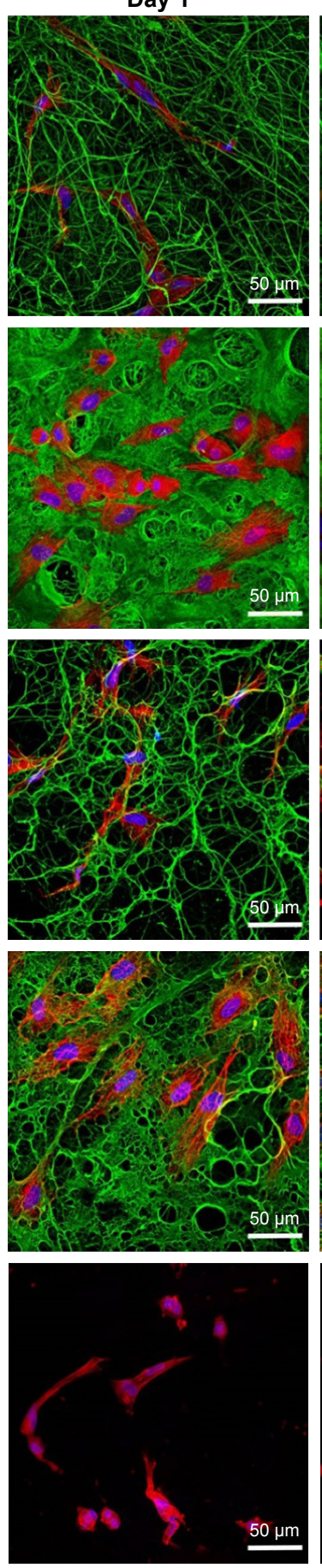

With fibroblasts

Day 3
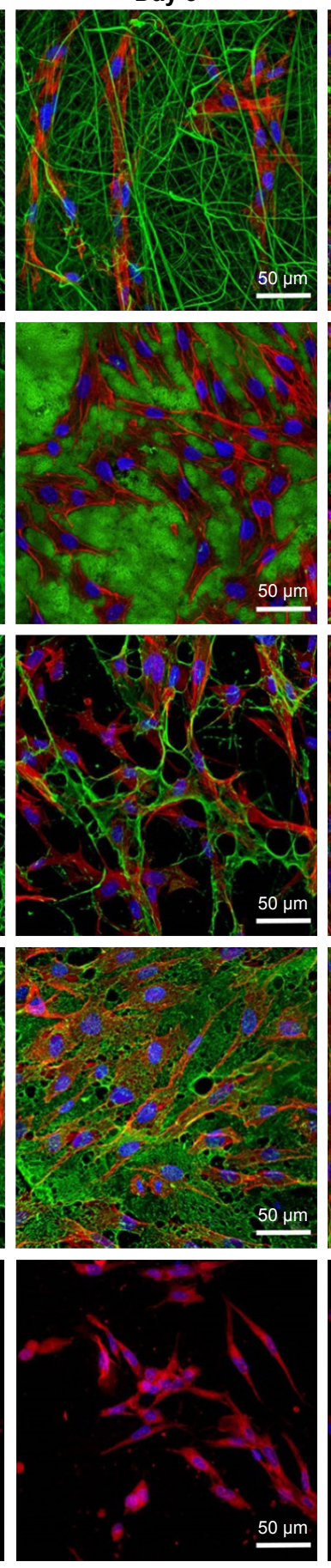

Day 7
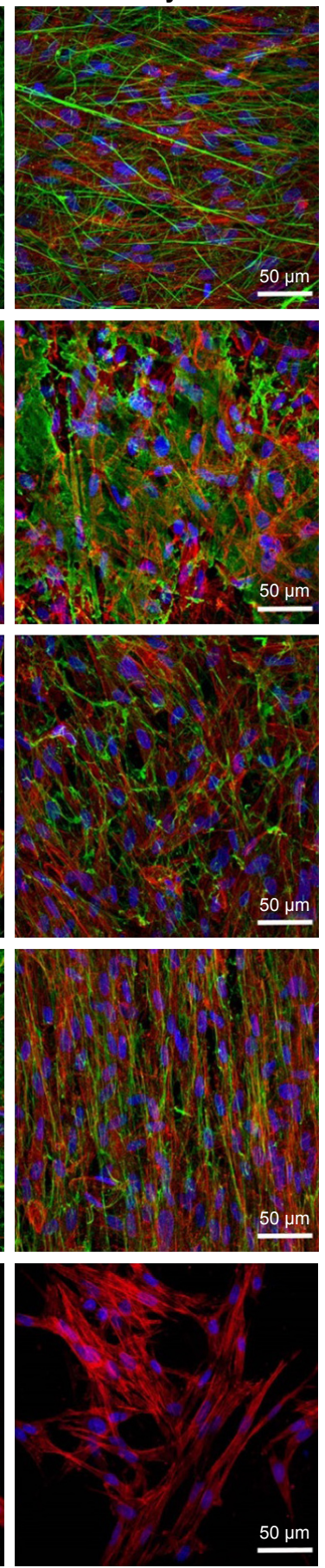

Figure I Immunofluorescence staining of two different types of fibrin nanocoatings on polylactic acid membrane (column I, in PBS) and the shape of human dermal fibroblasts on the fibrin nanocoatings (columns 2-4) on days I, 3, and 7 after cell seeding. The membrane with the fibrin nanocoating covering only individual fibers (FI), with fibrin covering individual fibers and forming a mesh on the surface of the membrane (F2), and Fn adsorbed on fibrin (+Fn). A noncoated membrane (pristine) was used as a control sample. Fibrin and Fn nanocoatings were stained with primary and secondary antibodies (Alexa 488 - green). The cells were stained with phalloidin-tetramethylrhodamine (actin cytoskeleton - red) and with Hoechst \#33258 (cell nuclei - blue). Leica TCS SPE DM2500 confocal microscope, magnification 40×/I.I5 numerical aperture oil.

Abbreviation: Fn, fibronectin.

adhered fibroblasts mimicked well the morphology of the Fn mesh (Figure 2B and Figure 1, F2+Fn).

The enhancing effect of the fibrin mesh (F2) and of the Fn mesh (F2+Fn) on cell growth was evident mainly in the later cell cultivation intervals (Figure 1). On day 3 after cell seeding, several cell mitoses were observed on membranes with F2 and F2+Fn nanocoatings, but the cells remained mostly in their polygon-like shape (Figure 1). On membranes 
Table I Mechanical puncture testing of wet membranes

\begin{tabular}{lllll}
\hline & & Pristine & FI & F2 \\
\hline$k$ & $\mathrm{~N} / \mathrm{mm}$ & $0.494 \pm 0.17$ & $0.486 \pm 0.1 \mathrm{I}$ & $0.598 \pm 0.28$ \\
$x_{\text {Fmax }}$ & $\mathrm{mm}$ & $3.344 \pm 0.10^{*}$ & $3.042 \pm 0.23^{*}$ & $3.282 \pm 0.12$ \\
$F_{\text {max }}$ & $\mathrm{N}$ & $1.234 \pm 0.42$ & $1.116 \pm 0.18$ & $1.554 \pm 0.79$ \\
$W$ & $\mathrm{~J}$ & $4.609 \pm \mathrm{I} .69$ & $3.558 \pm 0.56$ & $4.883 \pm 2.50$
\end{tabular}

Notes: The values are stated as mean \pm SD. $k$ represents the stiffness of the membrane, $F_{\max }$ is the detected maximum force, $x_{F \max }$ is the membrane extension at the maximum force, and $W$ is the work necessary to completely tear the membrane. $\mathrm{FI}$ - the membrane with fibrin covering only individual fibers. F2 - the membrane with fibrin covering individual fibers and forming a mesh on the surface of the membrane. A pristine noncoated membrane was used as a control material. Each group contained six independent samples and $p$-value returned by one-way analysisof-variance test for $k=0.025, x_{\text {Fmax }}=0.599, F_{\text {max }}=0.376$, and $W=0.417$. *A significant difference was found between the pristine and FI groups by the post hoc Tukey's honest significant difference test.

with F1 and F1+Fn nanocoatings, the cells proliferated less, but their spindle-like shape enabled them to migrate into the fibrin-coated PLA membrane (Figure 1). On day 7 after cell seeding, the fine fibrin (F2) and Fn meshes (F2+Fn) were almost degraded, and the fibroblasts penetrated into the deeper layers of the PLA membrane. After 1 week, the Fn mesh on the membranes with F1+Fn and F2+Fn nanocoatings was completely replaced by the extracellular Fn produced by the fibroblasts (Figure 1). On the noncoated membranes, which were used as a control material, the fibroblasts were mostly round and were not strongly adhered, in all three cultivation intervals (Figure 1, pristine).

The cell mitochondrial activity (measured by an MTS assay) was used as an indirect method for estimating the cell proliferation. The metabolic activity of human dermal fibroblasts was measured on days 1, 3, and 7 after cell seeding (Figure 3). In all intervals, it was detected that the fibroblast mitochondrial activity was significantly higher on the fibrin-coated membranes than on the pristine (noncoated) membranes, which is in accordance with our previous works. ${ }^{12,13}$ The most apparent differences between the
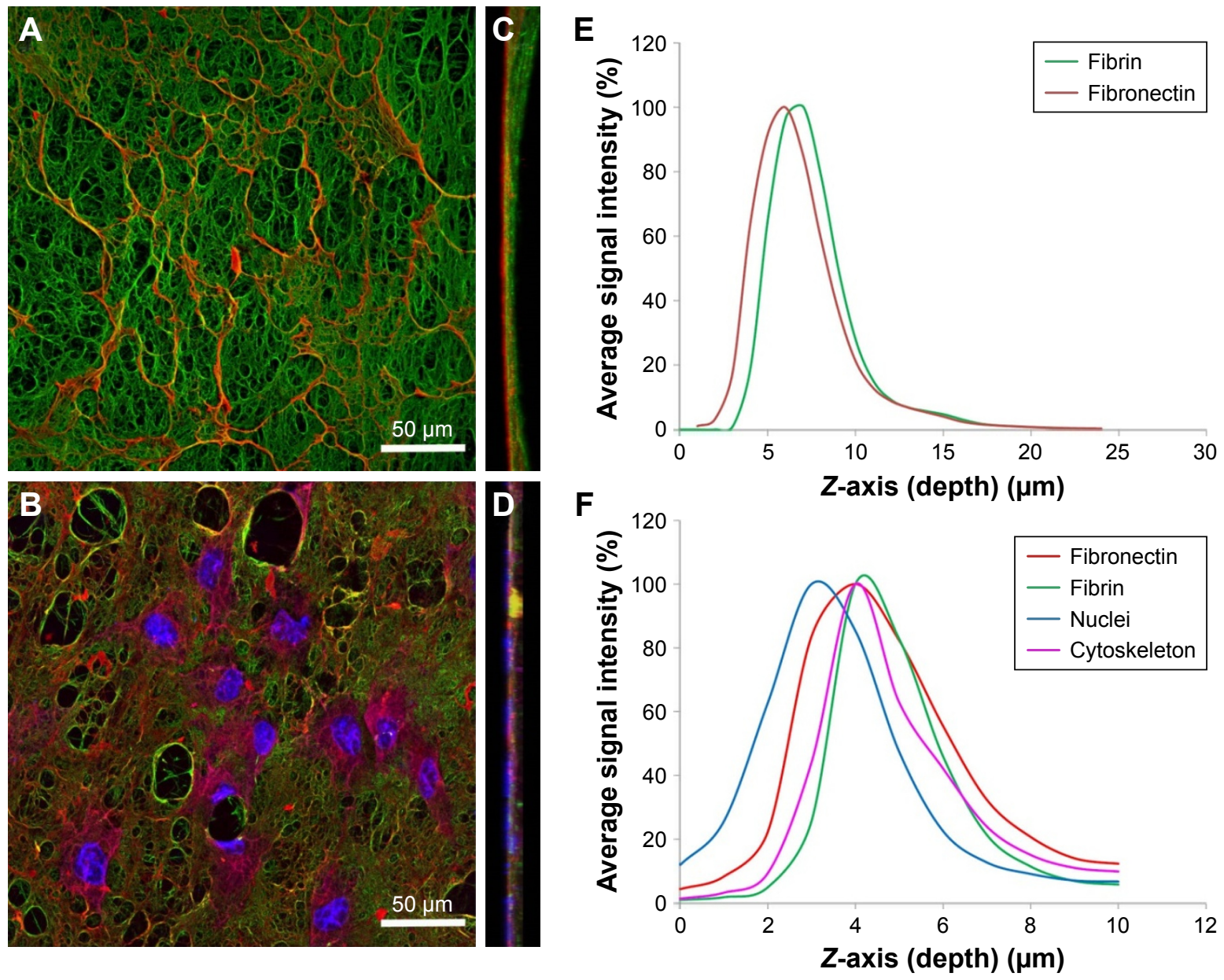

Figure 2 The signal intensity profiles of immunofluorescence stained fibrin mesh (F2 nanocoating) with attached fibronectin, without (A) or with (B) human dermal fibroblasts on day I after cell seeding. Fibrin and fibronectin were stained with primary and secondary antibodies (fibrin - Alexa 488 - green and fibronectin - Alexa 633 - red). The cells were stained with phalloidin-tetramethylrhodamine (actin cytoskeleton - magenta) and with Hoechst \#33258 (cell nuclei - blue). Maximum intensity projection - front view $(\mathbf{A}, \mathbf{B})$ and side view (C, D). The function plots (E, F) represent the average image plane intensity in a given color channel versus the $z$-axis (depth) of a confocal z-stack image. Leica TCS SPE DM2500 confocal microscope, magnification 40×/I.I5 NA oil, and Leica TCS SP8, objective HCX PL APO, magnification 40×/I.25-0.75 NA Oil CS. Abbreviation: NA, numerical aperture. 


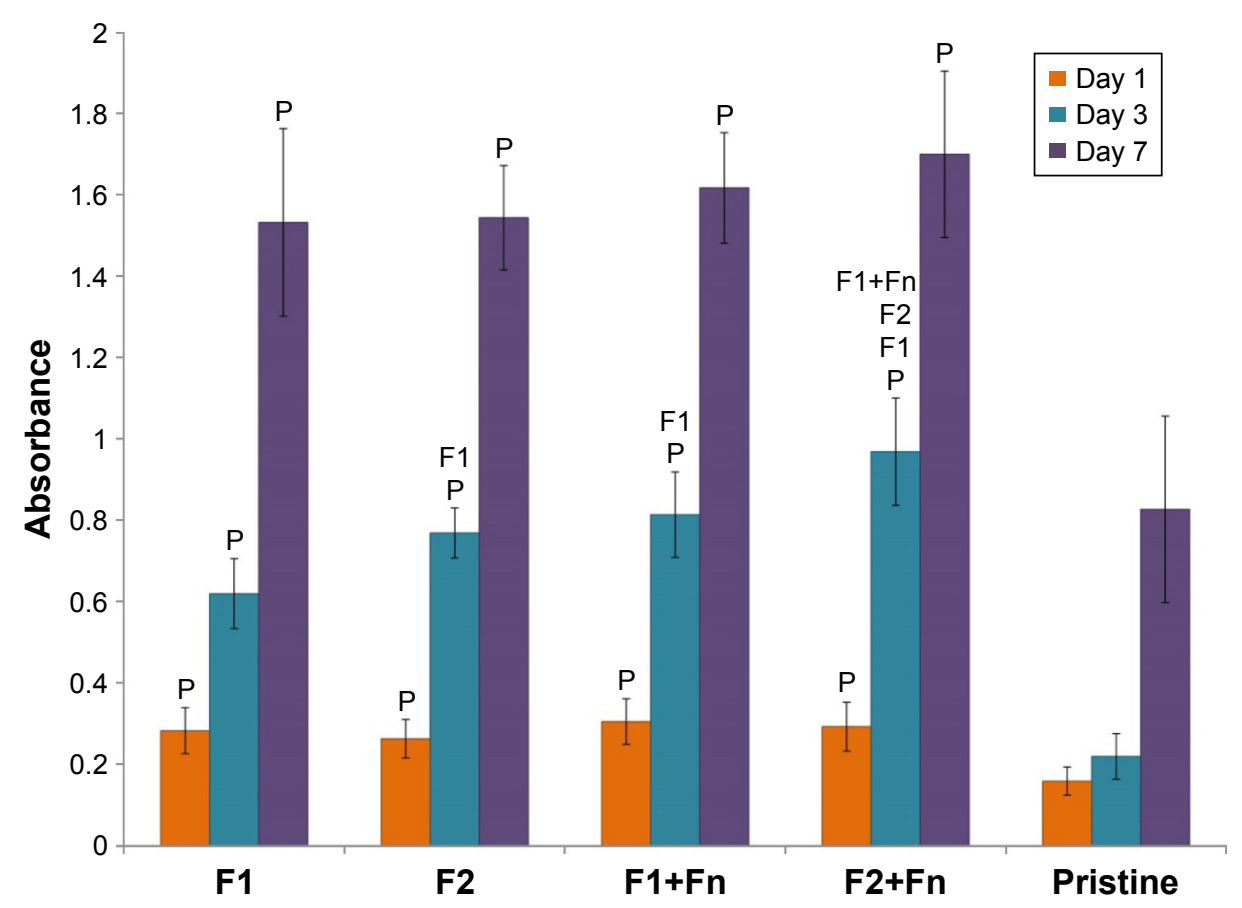

Figure 3 Mitochondrial activity of human dermal fibroblasts measured by MTS assay on days I, 3, and 7 after cell seeding on a polylactic acid membrane. The membrane with fibrin covering only individual fibers (FI), with fibrin covering individual fibers and forming a mesh on the surface of the membrane (F2), and Fn adsorbed on fibrin (+Fn). A noncoated membrane (pristine) was used as a control sample. Arithmetic mean \pm SD from nine measurements made on three independent samples for each experimental group and time interval. The experiment was performed three times. Statistical significance $(p \leq 0.05)$ evaluated using analysis of variance with Tukey's method is displayed above each experimental group, indicated by the abbreviation of the group or by $\mathrm{P}$ representing pristine.

Abbreviation: Fn, fibronectin.

types of fibrin nanocoatings of the cells were observed on day 3 after cell seeding. The cell mitochondrial activity was significantly higher on samples with a fibrin mesh (F2 and $\mathrm{F} 2+\mathrm{Fn}$ ) than on samples with $\mathrm{F} 1$ and $\mathrm{F} 1+\mathrm{Fn}$ nanocoatings (Figure 3). Fn attached to the fibrin significantly increased the cell mitochondrial activity (Figure 3, F1+Fn, F2+Fn). The significantly highest fibroblast mitochondrial activity was measured on the F2 nanocoating with an Fn mesh (Figure 3, F2+Fn). On days 1 and 7 after cell seeding, the cell mitochondrial activity was almost the same on all types of fibrin nanocoatings (Figure 3).

\section{ECM synthesis by human dermal fibroblasts}

The results of the relative mRNA expression from realtime PCR showed that the structure and the morphology of the fibrin nanocoatings also had a crucial impact on the expression of ECM proteins in the cells growing on these nanocoatings. The mRNA expression was measured in two cell culture intervals (on days 4 and 7 after cell seeding). On the basis of the previous studies, AA was added to the cell culture medium in order to enhance the expression and the formation of collagen I fibers. ${ }^{12}$ The mRNA expression of collagen I and Fn was significantly higher on F2 nanocoatings than on F1 nanocoatings (Figure 4A and B). Fn attached to the fibrin mesh $(\mathrm{F} 2+\mathrm{Fn})$ did not significantly influence the ECM expression (Figure 4A and B). However, Fn attached to $F 1$ nanocoatings $(F 1+F n)$ significantly increased the collagen I mRNA expression in both intervals (Figure 4A). On day 7, the collagen I expression on F1 nanocoatings with $\mathrm{Fn}(\mathrm{F} 1+\mathrm{Fn})$ was almost the same as the expression on the F2 nanocoatings (F2 and F2+Fn; Figure 4A). However, these differences were not observed in the expression of $\mathrm{Fn}$ mRNA (Figure 4B). The relative expression of collagen I was significantly higher on both types of fibrin nanocoatings than on the noncoated (pristine) PLA membranes (Figure 4A). The relative expression of $\mathrm{Fn}$ in the cells on $\mathrm{F} 1$ nanocoatings (F1 and F1+Fn) was almost the same as the relative expression on the pristine membranes. This pattern was observed in both cell culture intervals (Figure 4B).

All these results correlate with the immunofluorescence microscopy images in which the formation of collagen I and Fn fibers was visualized (Figures 5 and 6). Higher production of extracellular collagen I and Fn fibers was detected on PLA membranes with an F2 nanocoating than with an F1 nanocoating (Figures 5 and 6), which is in accordance with the measurements of relative mRNA expressions (Figure 4). 

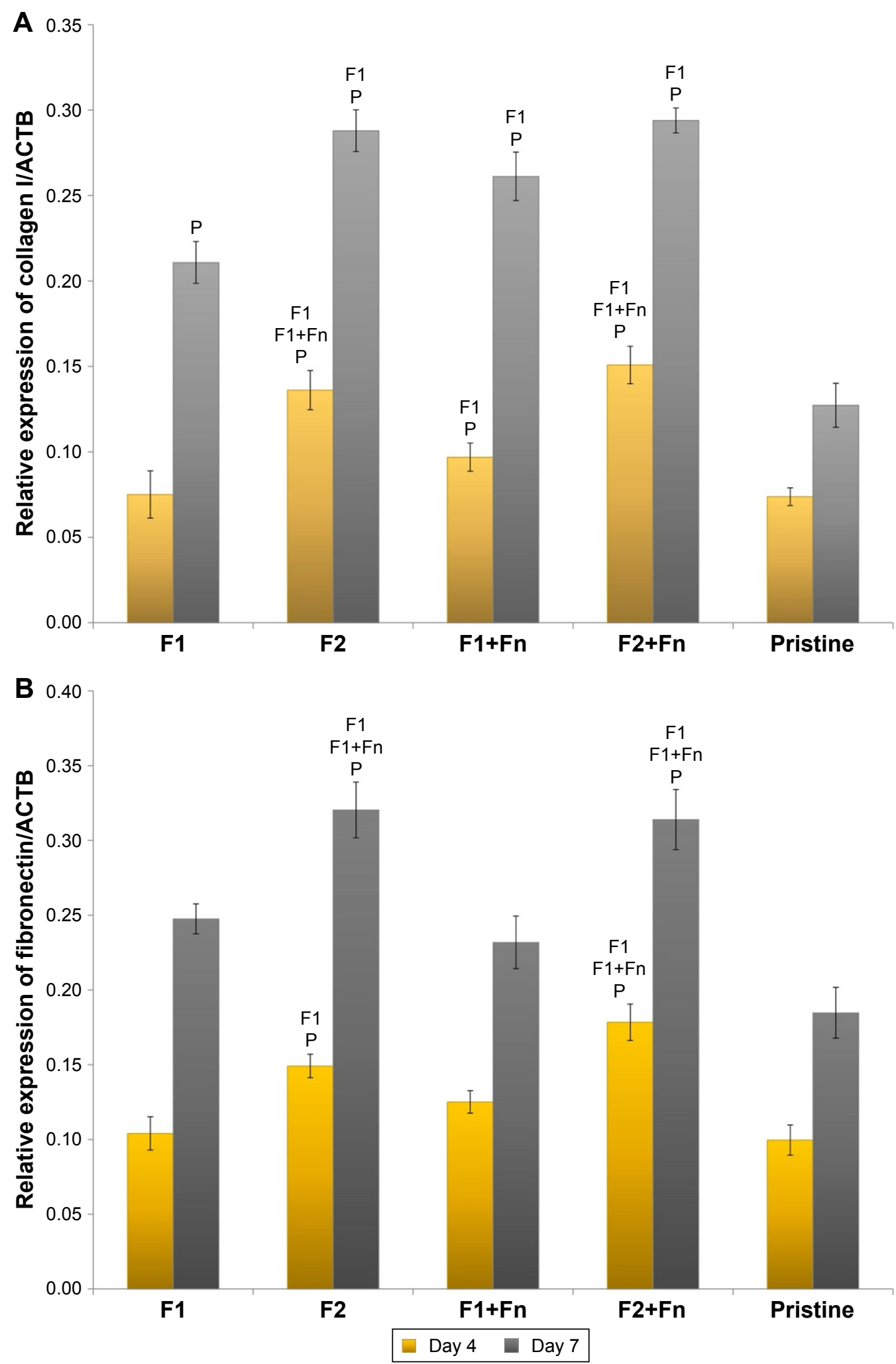

Figure 4 Relative expression of collagen I (A) and Fn (B) measured by real-time polymerase chain reaction in human dermal fibroblasts on days 4 and 7 after cell seeding on polylactic acid membranes with a fibrin structure. The membrane with fibrin covering individual fibers (FI), with fibrin covering individual fibers and forming a mesh on the membrane surface (F2), and Fn adsorbed on fibrin (+Fn). A noncoated membrane (pristine) was used as a control sample. ACTB was used as a reference gene. The arithmetic mean \pm SD was calculated for each experimental group and time interval from 10 measurements made in five independent experiments. Statistical significance $(p \leq 0.05)$ evaluated using analysis of variance with Tukey's method is displayed above each experimental group, indicated by the abbreviation of the group or by $P$ representing pristine.

Abbreviation: Fn, fibronectin. 

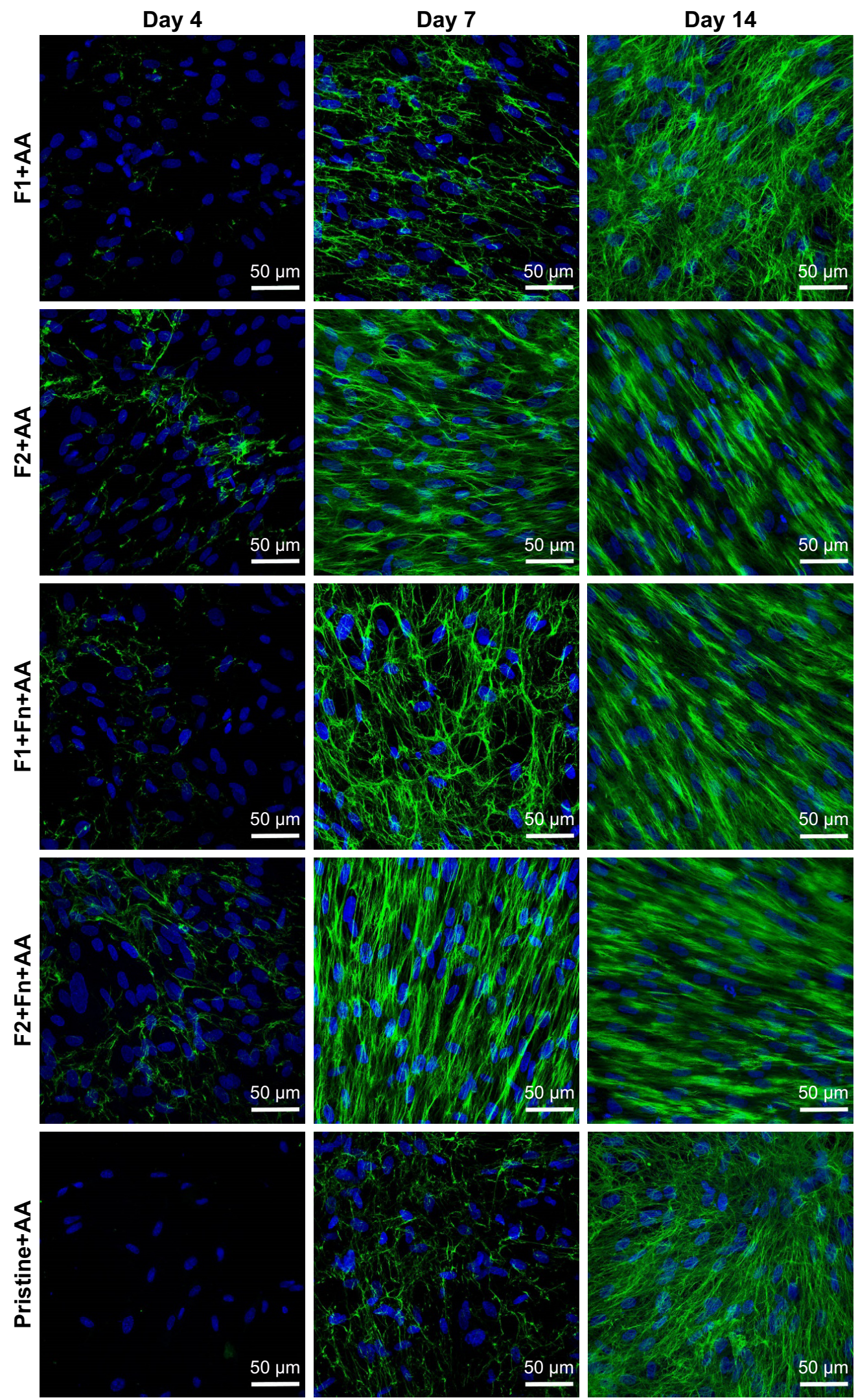

Figure 5 Immunofluorescence staining of extracellular collagen I fibers (green) produced by human dermal fibroblasts on polylactic acid membranes with two different types of fibrin nanocoatings on days 7, 10, and 14 after seeding. The membrane with fibrin covering individual fibers (FI), with fibrin covering individual fibers and forming a mesh on the membrane surface (F2), and Fn adsorbed on fibrin ( $+F n)$. A noncoated membrane (pristine) was used as a control sample. The cells were cultivated in the standard cell culture medium with AA. Cell nuclei stained with Hoechst \#33342 (blue). Leica TCS SPE DM2500 confocal microscope, magnification 40×/I.15 numerical aperture oil. Abbreviations: AA, 2-phospho-L-ascorbic acid trisodium salt; Fn, fibronectin. 

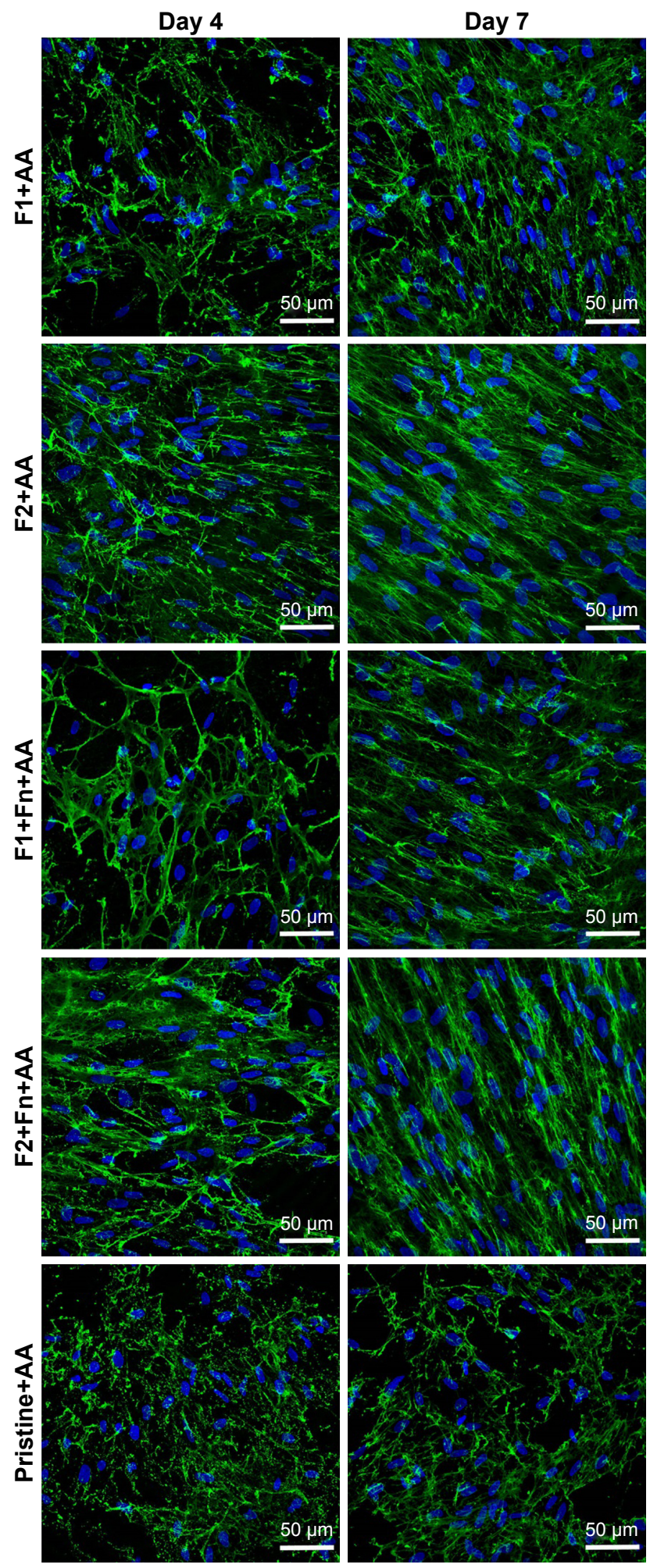

Figure 6 Immunofluorescence staining of extracellular Fn fibers (green) produced by human dermal fibroblasts on polylactic acid membranes with two different types of fibrin nanocoatings on days 4 and 7 after seeding. The membrane with fibrin covering individual fibers ( $\mathrm{FI})$, with fibrin covering individual fibers and forming a mesh on the membrane surface (F2), and Fn adsorbed on fibrin (+Fn). A noncoated membrane (pristine) was used as a control sample. The cells were cultivated in the standard cell culture medium with AA. Cell nuclei stained with Hoechst \#33342 (blue). Leica TCS SPE DM2500 confocal microscope, magnification 40x/I.I5 NA oil.

Abbreviations: AA, 2-phospho-L-ascorbic acid trisodium salt; Fn, fibronectin; NA, numerical aperture.

\section{Discussion}

The chemical and physical properties of the substrate for cell seeding are crucial for successful cell adhesion and growth. Many recent studies have discussed the influence of substrate properties (ie, surface wettability, electrical charge and conductivity, surface roughness and topography, substrate rigidity, and deformability) on the cell behavior and the colonization of a material with cells. ${ }^{16,20}$ Successful colonization of materials with desirable cell behavior, that is, cell adhesion, proliferation, migration, differentiation, and production of specific markers by cells as in a natural environment, is critical for the development of tissue substitutes. Skin substitutes are mostly fabricated from substrates based on natural biopolymers occurring in skin (mainly collagen) or during wound healing (fibrin). ${ }^{21}$ These proteins are favorable for cells because they support a desirable cell behavior. ${ }^{2}$ However, a protein scaffold in the form of a gel, a glue, or a sealant is mostly fragile, highly elastic, and deformable. These matrices are not stable, and they degrade quickly. Moreover, these material properties may lead to a collapse of the substrate under the traction forces of the adhering cells. ${ }^{22}$ Therefore, sufficient stability of the matrices could be achieved by depositing the biomolecules on an appropriate stronger carrier, for example, made of biodegradable polyesters such as PLA. This combined scaffold can provide stable mechanical support with appropriate properties for cell adhesion and growth. In our studies, we focus on the fabrication of PLA nanofibrous membranes coated with fibrin.

In this study, we prepared fibrin nanocoatings with different structures. The first nanocoating (F1) only covered the individual fibers in the membrane. The second nanocoating (F2) covered the individual fibers and, in addition, it formed a fine homogeneous nanofibrous mesh on surface of the membrane and filled the pores between the fibers. The different morphology and structure of $\mathrm{F} 1$ and $\mathrm{F} 2$ nanocoatings can be explained by the different mode of preparation for the two types of nanocoating. While the preparation of the F1 nanocoating included washing out the unbound thrombin with Tris buffer, the F2 nanocoating was prepared without washing out the thrombin. To the best of our knowledge, this phenomenon has not previously been described. These different modes of preparation probably controlled the amount of adsorbed thrombin on the membrane and the amount of subsequently bound fibrinogen to the active thrombin sites. In other words, the concentration of thrombin and fibrinogen adsorbed on the membrane could influence the formation of the fibrin coating. Previously published studies have revealed that the concentration of thrombin and fibrinogen had an 
impact on the formation of the fibrin network. A higher concentration of thrombin (above $1 \mathrm{U} / \mathrm{mL}$ ) induced the formation of thin fibrin fibers, while a lower concentration of thrombin (under $0.001 \mathrm{U} / \mathrm{mL}$ ) induced the formation of thick fibrin fibers. ${ }^{23,24}$ In our study, we used a highly concentrated thrombin $(2.5 \mathrm{U} / \mathrm{mL})$ solution, and we observed the formation of thin fibrin fibers, mainly in the case of the fibrin mesh (F2 nanocoating). The concentration of fibrinogen in solution during the adsorption influences the density, the orientation, and the accessibility of the individual fibrinogen domains of the surface-bound fibrinogen. At low fibrinogen surface density (under $20 \mu \mathrm{g} / \mathrm{mL}$ ), the majority of the molecules appear to be adsorbed side-on (lying on the surface). At high fibrinogen density (above $100 \mu \mathrm{g} / \mathrm{mL}$ ), the molecules are adsorbed end-on (standing on the surface). The experimental data suggest that the distal ends of the (adsorbed) fibrinogen molecules (containing polymerization sites) are oriented anti-parallel to each other, proving an off-axis location of the distal-end domains. ${ }^{25}$ Fibrinogen adsorbed from the initial lowly concentrated solution promoted the formation of a surface-attached fibrin network better than fibrinogen adsorbed from the highly concentrated solution. In our study, we prepared stable fibrin by initial adsorption of fibrinogen from a lowly concentrated solution $(10 \mu \mathrm{g} / \mathrm{mL})$, and then by incubation of the thrombin-activated surface with a highly concentrated fibrinogen solution $(200 \mu \mathrm{g} / \mathrm{mL})$. Riedel et al had previously shown that combining a lowly concentrated fibrinogen solution in the first step and a highly concentrated fibrinogen solution in the second step led to the formation of a well-developed fibrin network. The fibrin network grows on the surface because of the catalytic action of the surface-bound thrombin on the ambient fibrinogen solution. ${ }^{18}$ In the case of the F2 nanocoating, due to the thrombin nonwashing-out step, the concentration of thrombin adsorbed on the membrane was probably higher than in the case of the F1 nanocoating. This high concentration of thrombin may have enabled fibrinogen to bind in a greater amount in the second preparation step, leading to the formation of a fibrin mesh on the membrane. Moreover, Albala reported that fibrin sealants with high concentrations of fibrinogen tend to produce stronger clots. ${ }^{4}$ As was revealed by the membrane puncture testing performed in our study, the F2 nanocoating improves the mechanical properties of PLA membranes only slightly, while there was also quite high variability within the treated group. There was even less membrane extension with the F1 coating at maximum force, than with the pure PLA membrane (Table 1). In general, scaffolds fabricated from fibrin are usually mechanically weak, not self-supporting, and require reinforcement with mechanically more resistant natural or synthetic polymers, for example, hyaluronic acid, ${ }^{26}$ polyvinyl alcohol, ${ }^{27}$ or PLA. ${ }^{28}$

In this study, we have proved that fibrin deposited on PLA membranes strongly improves the cell adhesion, proliferation, and synthesis of ECM proteins (collagen I and Fn). This confirms the results from our previous works. ${ }^{12,13}$ As we have discussed, fibrin enabled cell adhesion by binding a specific fibrin molecule site by an adhesion receptor, mainly integrins of group $\beta_{1}$. Fibrin also further enhanced cell adhesion by attaching other cell adhesion-mediating proteins (eg, Fn, vitronectin, or collagen) from the serum supplement of the cell culture medium. ${ }^{1,12}$

This study has revealed the different behavior of dermal fibroblasts depending on the differences in the morphology of the fibrin nanocoating covering the membrane. The results showed that the F2 nanocoating (fibrin mesh) provided better support than the F1 nanocoating for the adhesion and proliferation of dermal fibroblasts. In addition, the shape of the adhered cells differed with the type of fibrin nanocoating. While the cells on the F1 nanocoating remained in a spindle-like shape, a polygon-like shape was typical for the cells cultivated on the F2 nanocoating. The spindle-like shape of fibroblasts seems to be more physiologic in the 3-D environment of natural skin dermis. ${ }^{29}$ However, a 2-D substrate may provide better support for the initial cell adhesion that results in homogeneous cell growth. Cells cultivated on flat substrates, for example, on PS culture dishes, mostly show faster initial adhesion and spreading than cells cultivated in 3-D matrices. However, 2-D substrates mostly do not enable the specific behavior of cells as in the natural 3-D system, mainly their migration and differentiation. ${ }^{30}$ The fine nanofibrous fibrin mesh of the F2 nanocoating resembled a 2-D surface, but its nanostructure mimicked the natural ECM, enabling faster and more successful adhesion of cells through adhesion receptors. ${ }^{17}$ The additional fibrin mesh in the F2 nanocoating filled the void spaces among the PLA fibers, and thus provided the cells with a larger area to adhere to. On this surface, the cells did not need to bridge the void spaces among the PLA fibers, and could utilize the entire lower part of their cytoplasmic membrane for adhesion through the cell adhesion receptors. This resulted in stronger specific mechanical and biochemical signals delivered to the cells. Moreover, the surface with the F2 coating could accommodate a higher number of cells. A similar improvement in cell behavior was observed in human osteoblast-like cells in cultures on PLGA and PLA nanofibers reinforced with diamond or hydroxyapatite nanoparticles. This led to reduction 
in the size of the void spaces among the nanofibers. ${ }^{31,32}$ In addition, fibroblasts are able to reorganize a nanostructured fibrin mesh, and this facilitates cell migration and synthesis of their own ECM proteins. ${ }^{33}$

The Fn attached to the fibrin nanocoating further improved the attachment of the cells and their subsequent proliferation. We showed a similar effect in our previous study. ${ }^{13} \mathrm{Fn}$ is known as a major adhesive protein with the RGD amino acid sequences bound by cells through adhesive receptors. ${ }^{34}$ The cells adhering to the fine nanofibrous Fn mesh formed secondarily on the F2 nanocoating followed the morphology of the mesh (Figure 2B). This observation suggests that $\mathrm{F} 2+\mathrm{Fn}$ nanocoating is the most similar to the natural environment.

On day 3 after cell seeding, we observed differences in the proliferation of dermal fibroblasts, depending on the type of nanocoating. The cell mitochondrial activity, which is also considered as an indirect indicator of cell proliferation, was higher on the F2 nanocoating than on the F1 nanocoating. Moreover, the Fn attached to the fibrin nanocoating led to improved cell proliferation. The highest cell metabolic activity was observed on the F2+Fn nanocoating. The fibrin mesh combined with the Fn mesh probably provided the best substrate for initial attachment of the fibroblasts, and this led to faster cell proliferation. We observed no differences in cell proliferation between samples on days 1 and 7 after cell seeding. After seeding, the cells concentrate physiologically on their attachment to the substrate, and they do not proliferate (lag phase). In later cell cultivation intervals (1 week), the cells reorganized and degraded the fine fibrin or the Fn mesh, and replaced it by their own ECM, mainly by collagen I and Fn. However, the samples with the F1 nanocoating mostly remained in non-reorganized form after 1 week of cell cultivation. From this point of view, the type of fibrin nanocoating did not affect the cell proliferation in later cell cultivation intervals. However, the mitochondrial activity of the cells was still significantly higher on the fibrin-coated membranes than on the noncoated membranes.

The morphology of the fibrin nanocoating also has a significant impact on the synthesis of ECM proteins collagen I and Fn. In our previous studies, we reported a positive effect of fibrin on the synthesis of collagen I and Fn. ${ }^{12,13}$ The present study has shown that the F2 nanocoating significantly increased the relative expression of collagen I and $\mathrm{Fn}$ and the formation of extracellular fibers of proteins on the material surface, whereas the F1 nanocoating showed mostly a similar effect to that of noncoated membranes. This pattern was especially observed on day 4 after cell seeding.
The fibroblasts contract physiologically and remodel the fibrin matrix formed after a skin injury, and finally replace it with their own synthesized ECM. ${ }^{6}$ If we compare F1 and F2 nanocoatings, it seems that the fine nanofibrous fibrin mesh of the F2 nanocoating was formed into a more physiologic structure, which enabled the fibroblasts to remodel it and replace it with their own ECM. Nevertheless, the relative expression of collagen I on the F1 nanocoating was enhanced by attaching Fn to the fibrin. It was shown that Fn is important for the production by fibroblasts of mature collagen fibrils and other extracellular proteins. ${ }^{35,36}$ Moreover, the Fn formed an additional nanofibrous mesh on top of the F1 nanocoating. As was mentioned above, the fine nanostructure seems to better mimic the natural ECM matrix. This can improve the healing process, and enable the fibroblasts to synthesize their own ECM in in vitro conditions.

\section{Conclusion}

The morphology of the fibrin nanocoating on PLA membranes can regulate the mechanical properties of the membrane and the behavior of dermal fibroblasts. A fine homogeneous fibrin mesh provides more support than fibrin coating of individual fibers for the adhesion, spreading, and proliferation of cells. The expression of ECM proteins was also enhanced by the fibrin mesh, and the highest ECM synthesis was stimulated on PLA membranes coated with a fibrin mesh with secondarily attached Fn. It can be concluded that fibrin nanocoating, especially in the form of a nanostructured homogeneous mesh, significantly accelerated the natural behavior of the cells. A homogeneous fibrin mesh can, therefore, be considered as a suitable coating for synthetic materials for subsequent application in skin tissue engineering.

\section{Acknowledgments}

This study was supported by the Grant Agency of the Czech Republic (grant 17-02448S) and by the Ministry of Education, Youth and Sports (grant LM2015062). Dr Denisa Stranska from InStar Technologies, Liberec, Czech Republic, is acknowledged for fabrication of the PLA nanofibrous membranes. Mr Robin Healey (Czech Technical University in Prague) is gratefully acknowledged for his language revision of the manuscript.

\section{Disclosure}

The authors report no conflicts of interest in this work.

\section{References}

1. Laurens N, Koolwijk P, de Maat MP. Fibrin structure and wound healing. J Thromb Haemost. 2006;4(5):932-939. 
2. Tracy LE, Minasian RA, Caterson EJ. Extracellular matrix and dermal fibroblast function in the healing wound. Adv Wound Care (New Rochelle). 2016;5(3):119-136.

3. Gailit J, Clarke C, Newman D, Tonnesen MG, Mosesson MW, Clark RA. Human fibroblasts bind directly to fibrinogen at RGD sites through integrin alpha(v)beta3. Exp Cell Res. 1997;232(1):118-126.

4. Albala DM. Fibrin sealants in clinical practice. Cardiovasc Surg. 2003; 11(Suppl 1):5-11.

5. Gugerell A, Pasteiner W, Nurnberger S, et al. Thrombin as important factor for cutaneous wound healing: comparison of fibrin biomatrices in vitro and in a rat excisional wound healing model. Wound Repair Regen. 2014;22(6):740-748.

6. Tuan TL, Song A, Chang S, Younai S, Nimni ME. In vitro fibroplasia: matrix contraction, cell growth, and collagen production of fibroblasts cultured in fibrin gels. Exp Cell Res. 1996;223(1):127-134.

7. Mazzone L, Pontiggia L, Reichmann E, Ochsenbein-Kolble N, Moehrlen U, Meuli M. Experimental tissue engineering of fetal skin. Pediatr Surg Int. 2014;30(12):1241-1247.

8. Zeng RX, He JY, Zhang YL, Liu XX, Zhang Y, Tang Q. Experimental study on repairing skin defect by tissue-engineered skin substitute compositely constructed by adipose-derived stem cells and fibrin gel. Eur Rev Med Pharmacol Sci. 2017;21(3 Suppl):1-5.

9. Han CM, Zhang LP, Sun JZ, Shi HF, Zhou J, Gao CY. Application of collagen-chitosan/fibrin glue asymmetric scaffolds in skin tissue engineering. J Zhejiang Univ Sci B. 2010;11(7):524-530.

10. Han HH, Jun D, Moon SH, Kang IS, Kim MC. Fixation of split-thickness skin graft using fast-clotting fibrin glue containing undiluted highconcentration thrombin or sutures: a comparison study. Springerplus. 2016;5(1):1902.

11. Gorodetsky R, Clark RA, An J, et al. Fibrin microbeads (FMB) as biodegradable carriers for culturing cells and for accelerating wound healing. J Invest Dermatol. 1999;112(6):866-872.

12. Bacakova M, Musilkova J, Riedel T, et al. The potential applications of fibrin-coated electrospun polylactide nanofibers in skin tissue engineering. Int J Nanomedicine. 2016;11:771-789.

13. Bacakova M, Pajorova J, Stranska D, et al. Protein nanocoatings on synthetic polymeric nanofibrous membranes designed as carriers for skin cells. Int J Nanomedicine. 2017;12:1143-1160.

14. Murad S, Grove D, Lindberg KA, Reynolds G, Sivarajah A, Pinnell SR. Regulation of collagen synthesis by ascorbic acid. Proc Natl Acad Sci U S A. 1981;78(5):2879-2882.

15. Park HJ, Ock SM, Kim HJ, et al. Vitamin C attenuates ERK signalling to inhibit the regulation of collagen production by LL-37 in human dermal fibroblasts. Exp Dermatol. 2010;19(8):E258-E264.

16. Bacakova L, Filova E, Parizek M, Ruml T, Svorcik V. Modulation of cell adhesion, proliferation and differentiation on materials designed for body implants. Biotechnol Adv. 2011;29(6):739-767.

17. Sun L, Stout DA, Webster TJ. The nano-effect: improving the long-term prognosis for musculoskeletal implants. J Long Term Eff Med Implants. 2012;22(3):195-209.

18. Riedel T, Brynda E, Dyr JE, Houska M. Controlled preparation of thin fibrin films immobilized at solid surfaces. J Biomed Mater Res A. 2009;88(2):437-447.
19. Schindelin J, Arganda-Carreras I, Frise E, et al. Fiji: an open-source platform for biological-image analysis. Nat Methods. 2012;9(7):676-682.

20. Bacakova L, Svorcik V. Cell colonization control by physical and chemical modification of materials. In: Kimura D, editor. Cell Growth Processes: New Research. New York: Nova Science Publishers, Inc.; 2008:5-56.

21. Vig K, Chaudhari A, Tripathi S, et al. Advances in skin regeneration using tissue engineering. Int J Mol Sci. 2017;18(4):789.

22. Engler A, Bacakova L, Newman C, Hategan A, Griffin M, Discher D. Substrate compliance versus ligand density in cell on gel responses. Biophys J. 2004;86(1 Pt 1):617-628.

23. Carr ME Jr, Hermans J. Size and density of fibrin fibers from turbidity. Macromolecules. 1978;11(1):46-50.

24. Wolberg AS. Thrombin generation and fibrin clot structure. Blood Rev. 2007;21(3):131-142.

25. Dyr JE, Tichý I, Jiroušková M, et al. Molecular arrangement of adsorbed fibrinogen molecules characterized by specific monoclonal antibodies and a surface plasmon resonance sensor. Sens Actuat B Chem. 1998; 51(1):268-272.

26. Lee F, Kurisawa M. Formation and stability of interpenetrating polymer network hydrogels consisting of fibrin and hyaluronic acid for tissue engineering. Acta Biomater. 2013;9(2):5143-5152.

27. Bidault L, Deneufchatel M, Vancaeyzeele C, Fichet O, Larreta-Garde V. Self-supported fibrin-polyvinyl alcohol interpenetrating polymer networks: an easily handled and rehydratable biomaterial. Biomacromolecules. 2013;14(11):3870-3879.

28. Law JX, Musa F, Ruszymah BH, El Haj AJ, Yang Y. A comparative study of skin cell activities in collagen and fibrin constructs. Med Eng Phys. 2016;38(9):854-861.

29. Fernandez-Madrid F, Noonan S, Riddle J. The "spindle-shaped" body in fibroblasts: intracellular collagen fibrils. J Anat. 1981;132(Pt 2): 157-166.

30. Duval K, Grover H, Han LH, et al. Modeling physiological events in 2D vs. 3D cell culture. Physiology (Bethesda). 2017;32(4):266-277.

31. Parizek M, Douglas TE, Novotna K, et al. Nanofibrous poly(lactideco-glycolide) membranes loaded with diamond nanoparticles as promising substrates for bone tissue engineering. Int J Nanomedicine. 2012;7:1931-1951.

32. Novotna K, Zajdlova M, Suchy T, et al. Polylactide nanofibers with hydroxyapatite as growth substrates for osteoblast-like cells. J Biomed Mater Res A. 2014;102(11):3918-3930.

33. Mazlyzam AL, Aminuddin BS, Fuzina NH, et al. Reconstruction of living bilayer human skin equivalent utilizing human fibrin as a scaffold. Burns. 2007;33(3):355-363.

34. Ruoslahti E, Pierschbacher MD. New perspectives in cell adhesion: RGD and integrins. Science. 1987;238(4826):491-497.

35. Sottile J, Hocking DC. Fibronectin polymerization regulates the composition and stability of extracellular matrix fibrils and cell-matrix adhesions. Mol Biol Cell. 2002;13(10):3546-3559.

36. Sethi KK, Yannas IV, Mudera V, Eastwood M, McFarland C, Brown RA. Evidence for sequential utilization of fibronectin, vitronectin, and collagen during fibroblast-mediated collagen contraction. Wound Repair Regen. 2002;10(6):397-408.
International Journal of Nanomedicine

\section{Publish your work in this journal}

The International Journal of Nanomedicine is an international, peerreviewed journal focusing on the application of nanotechnology in diagnostics, therapeutics, and drug delivery systems throughout the biomedical field. This journal is indexed on PubMed Central, MedLine, CAS, SciSearch ${ }^{\circledR}$, Current Contents ${ }^{\circledR} /$ Clinical Medicine,
Dovepress

Journal Citation Reports/Science Edition, EMBase, Scopus and the Elsevier Bibliographic databases. The manuscript management system is completely online and includes a very quick and fair peer-review system, which is all easy to use. Visit http://www.dovepress.com/ testimonials.php to read real quotes from published authors. 